\title{
Correlation, Entropy, and Information Transfer in Black Hole Radiation
}

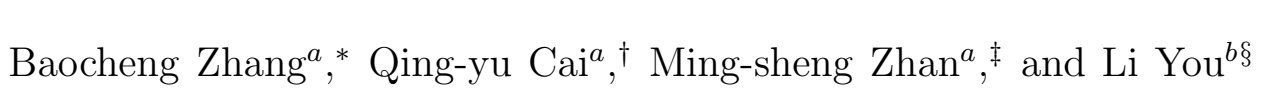 \\ ${ }^{a}$ State Key Laboratory of Magnetic Resonances and Atomic and Molecular Physics, \\ Wuhan Institute of Physics and Mathematics, \\ The Chinese Academy of Sciences, Wuhan 430071, China and \\ ${ }^{b}$ Department of Physics, State Key Laboratory of Low Dimensional Quantum Physics, \\ Tsinghua University, Beijing 100084, China
}

\begin{abstract}
Since the discovery of Hawking radiation, its consistency with quantum theory has been widely questioned. In the widely described picture, irrespective of what initial state a black hole starts with before collapsing, it eventually evolves into a thermal state of Hawking radiations after the black hole is exhausted. This scenario violates the principle of unitarity as required for quantum mechanics and leads to the acclaimed "information loss paradox". This paradox has become an obstacle or a reversed touchstone for any possible theory to unify the gravity and quantum mechanics. Based on the results from Hawking radiation as tunneling, we recently show that Hawking radiations can carry off all information about the collapsed matter in a black hole. After discovering the existence of information-carrying correlation, we show in great detail that entropy is conserved for Hawking radiation based on standard probability theory and statistics. We claim that information previously considered lost remains hidden inside Hawking radiation. More specifically, it is encoded into correlations between Hawking radiations. Our study thus establishes harmony between Harking radiation and the unitarity of quantum mechanics, which establishes the basis for a significant milestone towards resolving the long-standing information loss paradox. The paper provides a brief review of the exciting development on Hawking raidation. In addition to summarize our own work on this subject, we compare and address other related studies.
\end{abstract}

\footnotetext{
*Electronic address: zhangbc@wipm.ac.cn

${ }^{\dagger}$ Electronic address: qycai@wipm.ac.cn

${ }^{\ddagger}$ Electronic address: mszhan@wipm.ac.cn

$\S$ Electronic address: lyou@mail.tsinghua.edu.cn
} 


\section{INTRODUCTION}

Since the discovery of thermal radiation emissions from a black hole by Hawking [1, 2], the foundation of modern physics experiences a serious challenge as the thermal spectrum is inconsistent with the unitary principle of quantum mechanics [3]. A thermal spectrum implies information about matter collapsed into a black hole is lost in the process of black hole radiation. Many have attempted [4-11] to find a resolution but failed. This lack of a complete resolution makes the paradox of Hawking radiation an attractive topic up until now. In fact, each failed attempt for a resolution seems to have made the existence of this paradox more serious and attracted more interest. In particular, after the possibility that information about infallen matter may hide inside correlations between Hawking radiation and the internal states of a black hole was ruled out [11], it seemed either unitarity or Hawking's semiclassical treatment of radiation must break down.

An important reason for information loss in black hole radiation is usually attributed to the thermal spectrum, which was obtained by Hawking in his original calculation assuming a fixed background geometry without enforcing energy conservation [1 3] . A thermal spectrum rules out the existence of correlations among Hawking radiations, consequently the entropy for the whole system consisting of the black hole and its radiations must increase [12, 13]. However, the entropy for a closed system governed by quantum mechanics cannot increase. The thermal radiation from a black hole discovered by Hawking thus causes a serious conflict between general relativity and quantum mechanics.

A glimpse of hope was raised by the work [7] of Bekenstein, who noted that information leakage from a black hole is possible if the emitted radiations contain a subtle non-thermal correction instead of being pure thermal. This enlightened the path for a resolution to the paradox. A series of recent studies [14-16] claim that the paradox between black hole radiation and unitarity of quantum mechanics seem inevitable, even with the small correction to Hawking's leading order calculation. These discussions, however, can only support a judgement that a possible reason for the breakdown of unitarity based on the result of a semiclassical calculation of the black hole radiation lies at the assumption of a fixed spacetime background. Although analysis about whether a small non-thermal correction could save the lost information were made in these papers [14-16], their assumption of a fixed spacetime background made the physical mechanism from a small correction unclear and consequently 
the small corrections discussed in these studies are trivial.

Recently, Parikh and Wilczek [17] treated Hawking radiation as tunneling while enforcing energy conservation. Their method goes beyond a fixed spacetime background and include back reaction from the emission. As a result the emission spectrum obtained becomes nonthermal. Starting with this non-thermal spectrum, we recently visited and revisited the paradox of black hole information loss [18 20]. Correlations are discovered to exist among Hawking radiations for all types of black holes [19, 20]. Upon carefully evaluating the amount of information carried away in these correlations, we find that information is not lost and the entropy for the total system of a black hole plus its radiations is conserved, i.e., information coded into the correlations and carried away by the Hawking radiation is found to match exactly the amount previously claimed lost. Our conclusion thus restores consistency of Hawking radiation with the principle of unitarity for quantum mechanics concerning an isolated system.

In this brief review, we will introduce and summarize our recent results in a more logical and explicit approach. Several important elements required to understand the information loss paradox and our proposed resolution will also be discussed. The rest of this paper is organized as follows. We will discuss the reaction and the non-thermal radiation spectrum obtained using the method of Hawking radiation as tunneling in the second section. This is followed in the third section by the description of our earlier discovery of correlations among Hawking radiations and entropy conservation in the process of Hawking radiation as tunneling. With the total entropy conserved, a question naturally appears: why does a black hole has entropy? In the fourth section, we try to explain this by presenting the meaning of entropy for a black hole from the micro perspective of Hawking radiation. The fifth section concerns the transfer of information hidden in a black hole. Finally, we summarize and conclude in the sixth section.

\section{REACTION AND HAWKING RADIATION SPECTRUM}

The back reaction of emission is first considered in the calculation of black hole radiation as tunneling by Parikh and Wilczek [17]. The achievements and prospects for the tunneling method and its applications to Hawking radiation are documented in a recent topical review [21], which also include discussions on some of our recent work. 
In the tunneling method, the Schwarzschild coordinates

$$
d s^{2}=-\left(1-\frac{2 M}{r}\right) d t^{2}+\left(1-\frac{2 M}{r}\right)^{-1} d r^{2}+r^{2} d \Omega^{2}
$$

are usually transformed into the Painlevé coordinates

$$
d s^{2}=-\left(1-\frac{2 M}{r}\right) d t^{2}+2 \sqrt{\frac{2 M}{r}} d t d r+d r^{2}+r^{2} d \Omega^{2},
$$

which are stationary and non-singular at the horizon $r=2 M$ and present a background to define an effective vacuum state for a quantum field essentially for a free falling observer through the horizon. A second advantage for the Painlevé coordinates arises in the HamiltonJacobi method by the calculation of the temperature without the requirement of introducing a normalization [22]. In the Parikh-Wilczek tunneling method, the total mass is fixed while the mass of the black hole is allowed to fluctuate, and the particle of energy $E$ travels on its geodesics which is easily from the metric (2). Considering the self-gravitation effect, the metric becomes

$$
d s^{2}=-\left(1-\frac{2(M-E)}{r}\right) d t^{2}+2 \sqrt{\frac{2(M-E)}{r}} d t d r+d r^{2}+r^{2} d \Omega^{2},
$$

and the outgoing radial null geodesics of an emitted particle with energy $E$ is given by

$$
\dot{r}=1-\sqrt{\frac{2(M-E)}{r}},
$$

where the ingoing radial null geodesics is obtained by replacing 1 with -1 with the implicit assumption that $t$ increases towards the future.

Due to the infinite blueshift near the event horizon, the WKB approximation can be employed to calculate the tunneling probability. The calculation details can be found in the paper [17] and will not be reproduced here. We will instead only give the result for the tunneling probability

$$
\Gamma(E) \sim \exp \left[-8 \pi E\left(M-\frac{E}{2}\right)\right]=\exp (\Delta S),
$$

where $S=4 \pi M^{2}$ is the black hole's Bekenstein-Hawking entropy. This method of calculation enforces energy conservation, or the reaction from Hawking radiation. The emission spectrum is found to be non-thermal, dependent on the energy of tunneling particle. Although the tunneling mechanism is used, the black hole radiation is still understood as stemming 
from quantum fluctuations of virtual particles near the event horizon. After a pair of virtual particles is created at the outside of the black hole, the particle with negative energy tunnels into the black hole with probability $\Gamma$, while the particle with positive energy flies away and will be observed at infinity as black hole radiation. Since particles with negative energy might not tunnel into the black hole completely, the remainder are annihilated by their counterparts (particles with positive energy) at the outside of the black hole. This assures particles with positive energy fly away with the same tunneling probability $\Gamma$, which is also the probability for black hole radiation. Sometimes, tunneling are explained inside and near the event horizon of a black hole with a similar correspondence, but whether quantum fluctuations of the interior of a black hole is the same as that described by the common quantum field theory is unclear.

We note that in one of the final remarks of the paper [17], Parikh and Wilczek speculate about the possibility of information-carrying correlations in the non-thermal radiation spectrum (5). A recent paper [14], however, presents a theorem that states the opposite. It claims that for a traditional black hole or the Schwarzschild black hole, information about collapsed matter would be inevitably lost in the semiclassical consideration of Hawking radiation, even including the small corrections to the leading order calculation by Hawking. Otherwise other paradoxes like infinite entanglement caused by remnants would appear. While this theorem is self-consistent within its own frame of a fixed spacetime background, it is different from the considerations leading to the non-thermal spectrum which includes the reaction to the spacetime [23]. It is interesting to note that information loss paradox by a fixed spacetime background has caused a speculation for gauge-gravity duality [24] as a powerful tool to explore quantum gravity, even in anti-de Sitter space.

As alluded to in the above, we introduce in the following, Hawking radiation as tunneling invokes pair creation before tunneling and modifications to a fixed spacetime background using the back reaction from emission. The emission spectrum consequently becomes nonthermal. For the increased dimensionality of the interior Hilbert space of a black hole due to the in-falling particles with negative energy, one could assume the dimensionality of the whole Hilbert space remains unchanged in the radiation process. The creation of the pair outside the horizon and the annihilation of the particle inside the horizon are treated only as intermediate processes to accommodate for the change of an initial black hole into a reduced black hole plus radiation. Other discussions prefer to presume an initial Hilbert space as 
consisting of the black hole and its exterior [25, 26], which might be easier to arrive at a unitary process with a semiclassical thermal black hole radiation.

\section{CORRELATION AND ENTROPY CONSERVATION}

In order to resolve the paradox with the non-thermal spectrum (5), the first question we have to ask is whether there exist correlations among the non-thermal radiations. This same question was initially addressed by Parikh [27] and then by Arzano et al [28] with a negative answer, i.e., both found existence of no correlations. In our recent work, we find, however, that their answers are incorrect and correlations indeed exist among Hawking radiations from a non-thermal spectrum.

So what is the correct answer? For that, we go to the statistical theory. Given two statistical events, like two emissions of Hawking radiation, with their joint probability denoted by $p(A, B)$, the probabilities for each single emissions are given by $p(A)=\int p(A, B) d B$ and $p(B)=\int p(A, B) d A$. We then proceed with a simple check to see whether $p(A, B)=$ $p(A) \cdot p(B)$ holds true or not. If the equality sign holds true, no correlation exists between event $A$ and event $B$. The two events are independent. This is indeed the case when the emission spectrum is thermal. For the non-thermal spectrum of Eq. (5) , the two emissions are dependent, thus correlated. Alternatively, we can check if the conditional probability $p(B \mid A)=p(A, B) / p(A)$ of event $B$ to occur given that event $A$ has occurred is equal to the probability $p(B)[29]$.

As we have previously shown, when considering two subsequent emissions with energies $E_{1}$ and $E_{2}$, the tunneling probability for a particle of energy $E_{2}$ has to be treated carefully. Since we cannot assume in advance whether there exists correlation between the two emissions

or not, we have to get the two probabilities by taking the integral of their joint probability $\Gamma\left(E_{1}, E_{2}\right)$, i.e. $\Gamma\left(E_{1}\right)=\int \Gamma\left(E_{1}, E_{2}\right) d E_{2}$ and $\Gamma\left(E_{2}\right)=\int \Gamma\left(E_{1}, E_{2}\right) d E_{1}$. The joint probability refers to the two emissions with energies $E_{1}$ and $E_{2}$ occuring simultaneously, i.e., we have

$$
\Gamma\left(E_{1}, E_{2}\right)=\Gamma\left(E_{1}+E_{2}\right)
$$

which holds true for the non-thermal spectrum of Eq. (5) as energy conservation is enforced for its derivation within the treatment of Hawking radiation as tunneling. Then we get the 
two independent tunneling probabilities respectively as

$$
\begin{aligned}
& \Gamma\left(E_{1}\right)=\exp \left[-8 \pi E_{1}\left(M-\frac{E_{1}}{2}\right)\right], \\
& \Gamma\left(E_{2}\right)=\exp \left[-8 \pi E_{2}\left(M-\frac{E_{2}}{2}\right)\right] .
\end{aligned}
$$

Thus on one hand, we can affirm the existence of correlation by finding the conditional probability $\Gamma\left(E_{2} \mid E_{1}\right)=\frac{\Gamma\left(E_{1}, E_{2}\right)}{\Gamma\left(E_{1}\right)} \neq \Gamma\left(E_{2}\right)$, and on the other hand, due to $\Gamma\left(E_{1}, E_{2}\right) \neq$ $\Gamma\left(E_{1}\right) \cdot \Gamma\left(E_{2}\right)$, we can define a quantity to measure the correlation as in Ref. [28],

$$
C\left(E_{1}+E_{2} ; E_{1}, E_{2}\right)=\ln \Gamma\left(E_{1}, E_{2}\right)-\ln \left[\Gamma\left(E_{1}\right) \cdot \Gamma\left(E_{2}\right)\right]
$$

A simply calculation shows the correlation between two emissions is $8 \pi E_{1} E_{2}$; we can proceed to calculate the correlation between these two emissions with a third one of energy $E_{3}$, and the resulting correlation is found to be $8 \pi\left(E_{1}+E_{2}\right) E_{3}$; the total correlation among the three emissions $E_{1}, E_{2}$ and $E_{3}$ thus becomes $8 \pi E_{1} E_{2}+8 \pi\left(E_{1}+E_{2}\right) E_{3}$, which does not depend on the orders of the individual emissions. So for the non-thermal spectrum, the total correlation in a queue of Hawking radiations is summed up to

$$
C\left(M ; E_{1}, E_{2} \cdots E_{n}\right)=\sum_{n \geqslant 2} 8 \pi\left(E_{1}+E_{2}+\cdots+E_{n-1}\right) E_{n}=\sum_{i \geqslant j} 8 \pi E_{i} E_{j}
$$

where indices $i$ and $j$ take all possible values labeling individual emissions. The analogous correlation are found to vanish for a thermal spectrum.

Another proper description for the correlation appeals to the concept of the mutual information which is defined for a composite quantum system composed of subsystems $A$ and $B$ as

$$
S(A: B) \equiv S(A)+S(B)-S(A, B)=S(A)-S(A \mid B)
$$

where $S(A \mid B)$ is nothing but the conditional entropy [30]. This can be used to measure the total amount of correlations between any bi-partite system. To use the mutual information, we have to introduce the entropy for the discussed events here, i.e. the tunneling emission of particles. From our earlier analysis, the entropy taken away by the tunneling particle with energy $E_{i}$ after the black hole has emitted particles with a total energy $E_{f}=\sum_{j=1}^{i-1} E_{j}$ is given by

$$
S\left(E_{i} \mid E_{1}, E_{2}, \ldots, E_{i-1}\right)=-\ln \Gamma\left(E_{i} \mid E_{1}, E_{2}, \ldots E_{i-1}\right)
$$


Again $S\left(E_{i} \mid E_{1}, E_{2}, \ldots, E_{i-1}\right)$ denotes the conditional entropy that measures the entropy of emission $E_{i}$ given that the values of all the emitted particles with energies $E_{1}, E_{2}, \ldots$, and $E_{i-1}$ are known. Quantitatively, it is equal to the decrease of the entropy of a black hole with mass $M-E_{f}$ upon the emission of a particle with energy $E_{i}$. Such a result is also consistent with the thermodynamic second law for a black hole [31]: the emitted particles must carry entropies in order to balance the total entropy of the black hole and the radiation. When mutual information is applied to the emissions of two particles with energies $E_{1}$ and $E_{2}$, we have

$$
S\left(E_{2}: E_{1}\right) \equiv S\left(E_{2}\right)-S\left(E_{2} \mid E_{1}\right)=-\ln \Gamma\left(E_{2}\right)+\ln \Gamma\left(E_{2} \mid E_{1}\right) .
$$

Thus it is easy to find $S\left(E_{2}: E_{1}\right)=8 \pi E_{1} E_{2}$, which shows the correlation of Eq. (6) is exactly equal to the mutual information between the two sequential emissions.

The conditional entropy (8) can be used to calculate the total entropy for the radiations after a black hole evaporates, which is

$$
S\left(E_{1}, E_{2}, \cdots, E_{n}\right)=\sum_{i=1}^{n} S\left(E_{i} \mid E_{1}, E_{2}, \cdots, E_{i-1}\right),
$$

where $M=\sum_{i=1}^{n} E_{i}$ equals to the initial black hole mass due to energy conservation and $S\left(E_{1}, E_{2}, \ldots, E_{n}\right)$ denotes the joint entropy for all emissions. By a detailed calculation from Eq. (10), we previously show that the total entropy $S\left(E_{1}, E_{2}, \ldots, E_{n}\right)=4 \pi M^{2}$ exactly equals to the black hole's Bekenstein-Hawking entropy. This shows that the entropy of a black hole is indeed taken out by Hawking radiations, and the total entropy of all emitted radiations and the black hole is conserved during the black hole radiation process, which shows the consistency of Hawking radiation with the unitarity of quantum mechanics. Alternatively, this result can be understood by counting the number of ways a black hole can evaporate as in our earlier analysis [18] and in a later study by Israel and Yun [32]; i.e. the probability for evaporation of a black hole can be expressed as

$$
\Gamma\left(M \rightarrow M-E_{1} \rightarrow M-E_{1}-E_{2} \rightarrow \cdots \rightarrow 0\right)=e^{-S_{\mathrm{BH}}} .
$$

The number of ways for evaporation is then given by $N=e^{S_{\mathrm{BH}}}$ because $N \Gamma=1$, which provides an easy interpretation of the Bekenstein-Hawking entropy $S_{\mathrm{BH}}$ in terms of the number of modes for evaporation. In particular, at each step, the tunneling probability manifests itself as a quantum transition probability, i.e. $\Gamma \sim \frac{e^{S_{\text {fianl }}}}{e^{S_{\text {initial }}}}$, up to leading order. 


\section{ENTROPY}

In the previous section, we briefly reviewed our earlier results that there exist correlations among Hawking radiations treated as quantum tunneling. In the radiation process the total entropy for a black hole and its radiations is unchanged, which thus establishes its consistency with the unitarity of quantum mechanics. Although an interpretation of the BekensteinHawking entropy is provided in terms of the number of modes for a black hole's evaporation, the specific meaning of the entropy referred to Hawking radiation remains unclear from the discussions in the above. This puzzling point was coincidentally treated by us in another earlier study [33], where we interpret the above discussed entropy in terms of the uncertainty about the information of the precollapsed configurations of a black hole's forming matter, the self-collapsed configurations and the inter-collapsed configurations. Our interpretation can be applied to several relevant circumstances, including the formation of a black hole, the black hole coalescence, and a common matter dropped into a black hole.

Our interpretation can be presented using an explicit expression, i.e. the entropy carried away by an emitted particle with energy $E$,

$$
S(E)=8 \pi E(M-E)+\left(4 \pi E^{2}-S_{0}\right)+S_{0},
$$

where the entropy is partitioned into three parts with $S_{0}$ referring to the precollapsed configuration which reveals the information about the matter that will collapse into a black hole, $\left(4 \pi E^{2}-S_{0}\right)$ about self-collapsed configuration which reveals the inaccessible information about how to collapse, and the correlation or the partial information $8 \pi E(M-E)$ about inter-collapsed configuration which reveals that the inaccessible information about the interaction among different collapsed holes. Maybe it is better to state the entropy in another way, i.e. it means that the tunneling particle carries away all information about its precollapsed configuration, the self-collapsed configuration, and the inter-collapsed configuration.

The above partition spells out what we interpret the meaning of the Bekenstein-Hawking entropy from the radiations. Initially this insight was gained from observing a hypothetical collision of two Schwarzschild black holes with respective masses $m_{1}$ and $m_{2}$. Their respective entropies are $4 \pi m_{1}^{2}$ and $4 \pi m_{2}^{2}$. If the two black holes collide and form a new black hole with mass $m=m_{1}+m_{2}$ as required from energy conservation, the entropy for the new black hole

becomes $S_{m}=4 \pi\left(m_{1}+m_{2}\right)^{2}=4 \pi m_{1}^{2}+4 \pi m_{2}^{2}+8 \pi m_{1} m_{2}$ which is not equal to the sum of the entropies of the two initial black holes. This means correlations generated by gravitational 
interaction arise and are hidden inside the newly formed black hole and the exterior observer cannot obtain any information about them. On the other hand, when the two black holes collide and coalesce into one, gravitational waves will usually be emitted in the process. Is it possible that gravitational radiations carry away the amount of information related to the term $8 \pi m_{1} m_{2}$ as constrained by entropy conservation? Our earlier analysis [33] show that this is impossible. Actually Hawking radiation is probably the best messenger to carry away information locked inside a black hole according to our entropy partition (11). In particular, we have shown earlier that the information carried away does not include any redundant contents by considering a queue of emissions ordered according to $E_{1}, E_{2}, \cdots, E_{n-1}$, and $E_{n}$. The entropy for the first emission with an energy $E_{1}$ is

$$
S\left(E_{1}\right)=8 \pi E_{1}\left(M-E_{1}\right)+\left(4 \pi E_{1}^{2}-S_{01}\right)+S_{01}
$$

where the term $8 \pi E_{1}\left(M-E_{1}\right)$ includes all correlations between the particle with energy $E_{1}$ and all other particles with energies $E_{2}, \cdots, E_{n-1}$, and $E_{n}$. The entropy for the second emission with an energy $E_{2}$ given the first emission with an energy $E_{1}$ is

$$
S\left(E_{2} \mid E_{1}\right)=8 \pi E_{2}\left(M-E_{1}-E_{2}\right)+\left(4 \pi E_{2}^{2}-S_{02}\right)+S_{02}
$$

where we see that information about the interaction between the particles with energies $E_{1}$ and $E_{2}$ is taken out by the first emission. For the second emission, we therefore must subtract the quantity $8 \pi E_{1} E_{2}$ from the information it will take out. Analogously, for the entropy of the third emission with energy $E_{3}$, we find

$$
S\left(E_{3} \mid E_{1}, E_{2}\right)=8 \pi E_{3}\left(M-E_{1}-E_{2}-E_{3}\right)+\left(4 \pi E_{3}^{2}-S_{03}\right)+S_{03},
$$

again the correlations between the third emission $E_{3}$ with the first two of energies $E_{1}$ and $E_{2}$ need to be subtracted. Summing up the three entropies, we find there exists no redundant information or entropy

$$
\begin{aligned}
& S\left(E_{1}\right)+S\left(E_{2} \mid E_{1}\right)+S\left(E_{3} \mid E_{1}, E_{2}\right) \\
& =4 \pi\left(M-E_{1}-E_{2}-E_{3}\right)^{2}-4 \pi M^{2} \\
& =\Delta S_{\mathrm{BH}} .
\end{aligned}
$$

A step by step follow up calculation show that according to our suggested partition (11) not only that Hawking radiations carry with themselves all information with no loss or 
redundance. Thus it provides a self-consistent interpretation for the entropy of a black hole. It implies to an exterior observer, there exists an uncertainty or information about the back hole's precollapsed configuration, its self-collapsed configuration, and inter-collapsed configuration.

For the final radiation, there still remains some indefiniteness in the process of Hawking radiation as tunneling, since its entropy is given by

$$
S\left(E_{n} \mid E_{1}, E_{2} \cdots, E_{n-1}\right)=4 \pi E_{n}^{2}
$$

which is precisely the same as for a black hole with mass or energy $E_{n}$. This shows the final emission is really equivalent to no emission or emit itself. In other words, the Hawking radiation as tunneling cannot give any better description for the final emission than before, which can be compared to the views of considering the final black hole as a fundamental particle [34, 35] or a stable remnant [28, 36].

A second puzzling question contrasts how the entropy for an ordinary matter, which could essentially take any value, changes into a fixed value $4 \pi E^{2}$ after transformed into a black hole of an equal energy $E$ ? A clear decisive answer remains lacking here. However, we can shed some light on its answer using a conjecture by some physicists [37-40], which claims black holes have the maximum possible entropy of any object of equal size and as such makes them the likely end points of all entropy-increasing processes. For a clearer interpretation about the black hole entropy, we need a better description for the state of the black hole's interior.

\section{INFORMATION TRANSFER}

In the above sections, we have presented formally a mechanism about how to preserve unitarity in the process of Hawking radiation as tunneling, with the discovery of correlation among radiations from a non-thermal spectrum to balance the otherwise ever increasing entropies of Hawking radiations. Although we have pointed out implicitly that information can be taken out by Hawking radiation, the explicit mechanism for transfer (e.g. how information is encoded and decoded in the process of black hole collapse and radiation) is still unclear. In this section, we will give two examples to show that information hidden in a black hole can indeed be carried away by correlations among Hawking radiations, irrespective 
what the specific transfer mechanism is.

Our first example concerns Hawking radiation as tunneling through a quantum horizon [19,41]. The tunneling probability was already given [28] for a general spherically symmetric system in the ADM form [42] by referencing to the first law of black hole thermodynamics $d M=\frac{\kappa}{2 \pi} d S$,

$$
\Gamma \sim\left(1-\frac{E}{M}\right)^{2 \alpha} \exp \left[-8 \pi E\left(M-\frac{E}{2}\right)\right]=\exp (\Delta S),
$$

where $S=\frac{A}{4}+\alpha \ln A$ is the entropy derived by directly counting the number of micro-states with string theory and loop quantum gravity [28]. The coefficient $\alpha$ is negative in loop quantum gravity [43]. Its sign remains uncertain in string theory, depending on the number of field species in the low energy approximation [44]. For $\alpha>0$, we find $\Gamma \rightarrow 0$ when $E \rightarrow M$, but $S(M-E) \rightarrow \infty$. This causes difficulty in explaining the origin of an exponentially growing entropy when the black hole vanishes. However, qualitatively, this actually can be understood within the picture of Hawking radiation from a black hole. In the limit of $\Gamma(M)$ $=0$, the tunneling energy approaches the mass of the black hole, and the tunneling becomes slower and slower while the time to exhaust a black-hole approaches infinite. This infinity can also be obtained from other methods by using the Stefan-Boltzmann law as in Ref. [45]. For $\alpha<0$, it is known [36] that when the mass of a black hole approaches the critical mass $M_{c}$, no particles will be emitted. Using our previous analysis as presented in Eq. (10), one then obtain $S(M)-S\left(M_{c}\right)=\sum_{i} S\left(E_{i} \mid E_{1}, E_{2}, \cdots, E_{i-1}\right)$ or $S(M)=\sum_{i} S\left(E_{i} \mid E_{1}, E_{2}, \cdots, E_{i-1}\right)+$ $S\left(M_{c}\right)$. In Ref. [36], the mass $M_{c}$ is called the "zero point energy" of a black hole that is similar to a black hole remnant because it does not depend on the initial black hole mass. We showed that even with such a remnant, the total entropy remains conserved when information carried away by correlations are correctly included. Thus the unitarity remains true when the classical horizon is replaced by a quantum one for Hawking radiation.

More significantly, we want to affirm whether information about quantum gravity corrections or about $\alpha$ is taken out by Hawking radiations. This can be confirmed through the correlation

$$
C\left(E_{1}+E_{2} ; E_{1}, E_{2}\right)=8 \pi E_{1} E_{2}+2 \alpha \ln \frac{M\left(M-E_{1}-E_{2}\right)}{\left(M-E_{1}\right)\left(M-E_{2}\right)} \neq 0 .
$$

which shows that the information about quantum gravity effect is actually carried away if quantum gravity effects indeed exist in the interior of a black hole. 
The second example concerns noncommutative space [20, 46]. In order to include the noncommutative space effect in gravity, one has to change the mass of a gravitating object. The usual definition of mass density in commutative space is expressed in terms of Dirac delta function, but in noncommutative space this form breaks down due to position-position uncertainty relations. It was shown [47, 48] that noncommutativity eliminates point-like structures in favor of smeared objects in flat spacetime. The effect of smearing is implemented by redefining the mass density by a Gaussian distribution of a minimal width $\sqrt{\theta}$ instead of the Dirac delta function. Here $\theta$ is the noncommutative parameter which is considered to be a small (Planck length) positive number and comes from the noncommutator of $\left[x^{\mu}, x^{v}\right]=i \theta^{\mu \nu}$ with $\theta^{\mu \nu}=\theta \operatorname{diag}\left[\epsilon_{1}, \cdots, \epsilon_{D / 2}\right]$. The constancy of $\theta$ is related to a consistent treatment of Lorentz invariance and unitarity [49].

Under the assumption of spatial noncommutativity, the background Painlevé coordinate becomes [20, 46, 50]

$$
d s^{2}=-\left(1-\frac{4 M}{r \sqrt{\pi}} \gamma\right) d t^{2}+2\left(1-\frac{4 M}{r \sqrt{\pi}} \gamma\right) \sqrt{\frac{\frac{4 M}{r \sqrt{\pi}} \gamma}{\left(1-\frac{4 M}{r \sqrt{\pi}} \gamma\right)^{2}}} d t d r+d r^{2}+r^{2} d \Omega^{2}
$$

where $\gamma \equiv \gamma\left(\frac{3}{2}, \frac{r^{2}}{4 \theta}\right)$ and the spacetime described by (19) is still stationary. Then a detailed calculation gives the tunneling rate,

$$
\Gamma \sim \exp \left[-8 \pi E\left(M-\frac{E}{2}\right)+16 \sqrt{\frac{\pi}{\theta}} M^{3} e^{\frac{M^{2}}{\theta}}-16 \sqrt{\frac{\pi}{\theta}}(M-E)^{3} e^{\frac{(M-E)^{2}}{\theta}}\right],
$$

where the result is obtained up to the leading order $\frac{1}{\sqrt{\theta}} e^{-M^{2} / \theta}$. With the same procedures presented in the third section, one can prove the total entropy is still conserved in the radiation process with the background metric (19). In particular, we find the statistical correlation between two emissions

$$
C\left(E_{1}+E_{2} ; E_{1}, E_{2}\right)=C\left(E_{1}, E_{2}, \theta\right) \neq 0
$$

which shows that information about the spatial noncommutativity labeled by the parameter $\theta$ will be carried out if space is indeed noncommutative.

A recent paper [51] studied information transfer mechanism by attempting to match a black hole's evolution unitarily. Due to the complexity of black hole physics, e.g. it remains unknown what dynamics is really responsible for the description of a black hole's interior and most physicists even believe that physics near the event horizon (about the Planck 
length away from the horizon) cannot be properly described with quantum field theory, no workable information transfer mechanism has been found capable of fitting in with the black hole evolution to the presumed being unitarity, especially after the paradox of "firewall" [52] was put forward. Nevertheless, it is found that our description for Hawking radiation is the same as the mechanisms called "subsystem transfer" [51], since within each step the sum of the remaining black hole's entropy and the entropy carried away by the outgoing particles is exactly equal to the initial entropy of the black hole. This not only satisfies the condition required by information transfer, i.e. the entropy for a black hole decreases, it also saturates the subadditivity inequality required by the subsystem transfer, although we didn't assume in advance the existence of external Hilbert space as was done in the Ref. [51]. Maybe Page's work [53] with the Hilbert space consisting of a black hole and its radiation subsystem is closer to our description. But in his model the final entropy of radiation subsystem is found to decrease to zero.

\section{CONCLUSION}

In this brief review, we have presented our earlier studies and conclusions regarding the black hole information loss paradox. After our discovery that the non-thermal spectrum of Parikh and Wilczek allows for the Hawking radiation emissions to carry off all information of a black hole, a natural question to ask is whether Hawking radiation is indeed non-thermal or not? Although the derivation of the non-thermal spectrum is based on solid physics, it remains to be confirmed experimentally or in observations. A recent analysis by us [54] show that the non-thermal spectrum can indeed be distinguished from the thermal spectrum by counting the energy covariances of Hawking radiations. The energy covariances actually measure the correlation among Hawking radiations. With the relatively low energy scale for quantum gravity and the large dimensions, the production of micro black holes and the observation of Hawking radiations has already been studied [55-60]. If Hawking radiations from a micro black hole were observed in an LHC experiment, our results show that it can definitely determine whether the emission spectrum is indeed non-thermal [54] or not. Thus it provides an avenue towards experimentally testing the long-standing "information loss paradox". On the other hand, as shown in the previous section, the correlation we discuss is also the carrier of information hidden in the interior of a black hole, so if the correlation is 
found experimentally, more information about some fundamental theories such as quantum gravity might be revealed simultaneously.

We conclude that our studies show the existence of correlations among Hawking radiations and the total entropy is conserved in the whole radiation process, which is consistent with unitarity of quantum mechanics. Our series of studies resolve the paradox of information loss at least formally, although the framework of Hawking radiation as quantum tunneling constrains our ability to explore the internal dynamics of a black hole. We have also made some remarks and comments to several recent researches on the subject, and found no inconsistencies with our results. Although our recent work widely supports the claim that Hawking radiation process is not in conflict with quantum mechanics at the semiclassical level, a more refined description for the radiation process remains to be constructed. Further study along this direction could possibly confront the emergence of new physics. Irrespective

of that, however, as we declared in a recent essay [61], which won the first prize in the 2013 Essay Competition of the Gravity Research Foundation, information conservation is fundamental for any isolated system, even for a black hole with its radiations being part of the system.

\section{ACKNOWLEDGEMENT}

Financial support from National Natural Science Foundation of China under Grant Nos. 11104324, 11374330, 11074283, 11227803, 11374176, and 91121005.

[1] Hawking S W. Black hole explosions? Nature (London) 1974, 248: 30-31

[2] Hawking S W. Particle creation by black holes. Comm Math Phys, 1975, 43: 199-220

[3] Hawking S W. Breakdown of predictability in gravitational collapse. Phys Rev D, 1976, 14: $2460-2473$

[4] Aharonov Y, Casher A, Nussinov S. The unitarity puzzle and Planck mass stable particles. Phys Lett B, 1987, 191: 51

[5] Krauss L M, Wilczek F. Discrete gauge symmetry in continuum theories. Phys Rev Lett, 1989, 62: $1221-1223$

[6] Preskill J. Do Black Holes Destroy Information? arXiv: hep-th/9209058. 
[7] Bekenstein J D. How fast does information leak out from a black hole? Phys Rev Lett, 1993, 70: 3680

[8] Kiefer C. Hawking radiation from decoherence. Class Quantum Grav, 2001, 18: L151

[9] Horowitz G T, Maldacena J. The black hole final state. J High Energy Phys, 2004, 02: 008

[10] Hawking S W. Information loss in black holes. Phys Rev D, 2005, 72: 084013

[11] Braunstein S L, Pati A K. Quantum Information Cannot Be Completely Hidden in Correlations: Implications for the Black-Hole Information Paradox. Phys Rev Lett, 2007, 98: 080502

[12] Zurek W H. Entropy Evaporated by a black hole. Phys Rev Lett, 1982, 49: 1683-1686

[13] Page D N. Comment on "Entropy Evaporated by a black hole". Phys Rev Lett, 1982, 50: 1013

[14] Mathur S D. The information paradox: a pedagogical introduction. Class Quantum Grav, 2009, 26: 224001

[15] Mathur S D, Plumberg C J. arXiv: 1101.4899v2.

[16] Mathur S D. What the information paradox is not. arXiv: 1108.0302v2.

[17] Parikh M K, Wilczek F. Hawking radiation as tunneling. Phys Rev Lett, 2000, 85: 5042

[18] Zhang B, Cai Q Y, You L, et al. Hidden messenger revealed in Hawking radiation: A resolution to the paradox of black hole information loss. Phys Lett B, 2009, 675: 98

[19] Zhang B, Cai Q Y, Zhan M S, et al. Entropy is conserved in Hawking radiation as tunneling: A revisit of the black hole information loss paradox. Annals of Physics, 2011, 326: 350

[20] Zhang B, Cai Q Y, Zhan M S, et al. Noncommutative information is revealed from Hawking radiation as tunneling. EPL, 2011, 94: 20002

[21] Vanzo L, Acquaviva G, Criscienzo R D. Tunneling methods and Hawking's radiation: achievements and prospects. Class Quantum Grav, 2011, 28: 183001

[22] Zhang B, Cai Q Y, Zhan M S. The temperature in Hawking radiation as tunneling. Phys Lett B, 2009, 671: 310

[23] Cai Q Y, Zhang B C, Zhan M S, et al. arXiv: 1210.2408

[24] Marolf D, Polchinski J. Gauge-Gravity Duality and the Black Hole Interior. Phys Rev Lett, 2013, 111: 171301

[25] Banks T. Holographic Space-Time: The Takeaway. arXiv: 1109.2435

[26] Giddings S B. Black holes, quantum information, and unitary evolution. Phys Rev D, 2012, 85: 124063

[27] Parikh M K. Energy Conservation and Hawking Radiation. arXiv: hep-th/0402166 
[28] Arzano M A, Medved J M, Vagenas E C. Hawking radiation as tunneling through the quantum horizon. J High Energy Phys, 2005, 0509: 037

[29] Gardiner C. Stochastic Methods: A Handbook for the Natural and Social Sciences. Springer Series in Synergetics, 2009, Spinger, NY

[30] Nielsen M A, Chuang I L. Quantum Computation and Quantum Information. Cambridge University Press, 2000, Combridge, UK

[31] Hawking S W. Black holes and thermodynamics. Phys Rev D, 1976, 13: 191-197

[32] Israel W, Yun Z. Band-aid for information loss from black holes. Phys Rev D, 2010, 82: 124036

[33] Zhang B, Cai Q Y, Zhan M S, et al. An interpretation for the entropy of a black hole. Gen Relativ Grav, 2011, 43: 797

[34] t'Hooft G. On the quantum structure of a black hole. Nucl Phys B, 1985, 256: 727-745

[35] Ha Y K. Are Black Holes Elementary Particles? arXiv: 0906.3549

[36] Xiang L. A note on the black hole remnant. Phys Lett B, 2007, 647: 207

[37] Bekenstein J D. Universal upper bound on the entropy-to-energy ratio for bounded systems. Phys Rev D, 1981, 23: 287

[38] Susskind L. The World as a hologram. J Math Phys, 1995, 36: 6377

[39] Bousso R. A covariant entropy conjecture. J High Energy Phys, 1999, 07: 004

[40] Flanagan E E, Marolf D, Wald R M. Proof of classical versions of the Bousso entropy bound and of the generalized second law. Phys Rev D, 2000, 62: 084035

[41] Chen Y X, Shao K N. Information loss and entropy conservation in quantum corrected Hawking radiation. Phys Lett B, 2009, 678, 131-134

[42] Kraus P, Wilczek F. Self-interaction correction to black hole radiance. Nucl Phys B, 1995, 433: 403

[43] Ghosh A, Mitra P. Log correction to the black hole area law. Phys Rev D, 2005, 71: 027502

[44] Solodukhin S N. Entropy of the Schwarzschild black hole and the string-black-hole correspondence. Phys Rev D, 1998, 57: 2410-2414

[45] Fabbri A, Perez A. Black hole evaporation in a thermalized final-state projection model. Phys Rev D, 2007, 75: 064009

[46] Banerjee R, Majhi B R, Samanta S. Noncommutative black hole thermodynamics. Phys Rev D, 2008, 77: 124035

[47] Smailagic A, Spallucci E. Feynman path integral on the noncommutative plane. J Phys A, 
2003 36: L467

[48] Smailagic A, Spallucci E. UV divergence free QFT on noncommutative plane. J Phys A, 2003 36: L517

[49] Smailagic A, Spallucci E. Lorentz invariance, unitarity in UV-finite of QFT on noncommutative spacetime. J Phys A, 2004 37: 1; Erratum-ibid., 2004, 37: 7169

[50] Nicolini P, Smailagic A, Spallucci E. Noncommutative geometry inspired Schwarzschild black hole. Phys Lett B, 2006, 632: 547

[51] Giddings S B, Shi Y. Quantum information transfer and models for black hole mechanics. Phys Rev D, 2013, 87: 064031

[52] Almheiri A, Marolf D, Polchinski J, et al. Black holes: complementarity or firewalls? J High Energy Phys, 2013, 02: 062

[53] Page D N. Average entropy of a subsystem. Phys Rev Lett, 1993, 71: 1291-1294

[54] Zhang B, Cai Q Y, Zhan M S, et al. Towards experimentally testing the paradox of black hole information loss. Phys Rev D, 2013, 87: 044006; Erratum, 2013, 88: 044901(E)

[55] Banks T, Fischler W. A model for high energy scattering in quantum gravity. arXiv: hep-th/9906038

[56] Emparan R, Horowitz G T, Myers R C. Black holes radiate mainly on the brane. Phys Rev Lett 85: 499

[57] Dimopoulos S, Landsberg G. Black holes at the Large Hadron Collider. Phys Rev Lett, 2001, 87: 161602

[58] Giddings S B, Thomas S. High energy colliders as black hole factories: The end of short distance physics. Phys Rev D, 2002, 65: 056010

[59] Meade P, Randall L. Black holes and quantum gravity at the LHC. J High Energy Phys, 2008, 05: 003

[60] CMS Collaboration, Search for microscopic black hole signatures at the Large Hadron Collider. Phys Lett B, 2011, 697: 434

[61] Zhang B, Cai Q Y, Zhan M S, et al. Information conservation is fundamental: recovering the lost information in Hawking radiation. IJMPD, 2013, 22: 1341014 